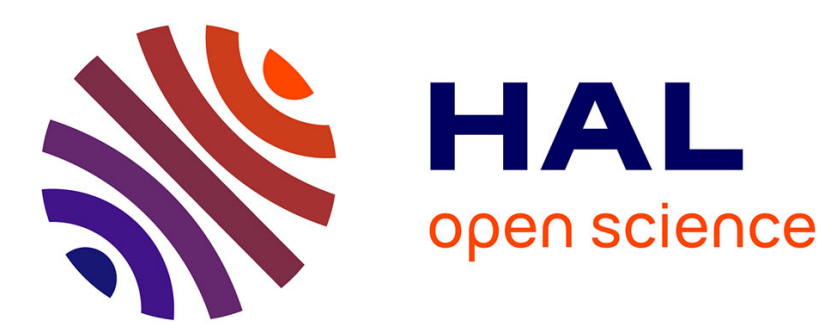

\title{
A Comparison of Active Classification Methods for Content-Based Image Retrieval
}

\author{
Philippe-Henri Gosselin, Matthieu Cord
}

\section{To cite this version:}

Philippe-Henri Gosselin, Matthieu Cord. A Comparison of Active Classification Methods for ContentBased Image Retrieval. International Workshop on Computer Vision meets Databases, ACM Sigmod, Jun 2004, France. pp.1. hal-00520318

\section{HAL Id: hal-00520318 https://hal.science/hal-00520318}

Submitted on 22 Sep 2010

HAL is a multi-disciplinary open access archive for the deposit and dissemination of scientific research documents, whether they are published or not. The documents may come from teaching and research institutions in France or abroad, or from public or private research centers.
L'archive ouverte pluridisciplinaire HAL, est destinée au dépôt et à la diffusion de documents scientifiques de niveau recherche, publiés ou non, émanant des établissements d'enseignement et de recherche français ou étrangers, des laboratoires publics ou privés. 


\section{A Comparison of Active Classification Methods for Content-Based Image Retrieval}

\author{
Philippe H. Gosselin \\ ETIS CNRS UMR-8051 \\ University of Cergy-Pontoise \\ ENSEA \\ 6, av. du Ponceau \\ 95014 Cergy-Pontoise, France \\ gosselin@ensea.fr
}

\author{
Matthieu Cord \\ ETIS CNRS UMR-8051 \\ University of Cergy-Pontoise \\ ENSEA \\ 6, av. du Ponceau \\ 95014 Cergy-Pontoise, France \\ cord@ensea.fr
}

\begin{abstract}
This paper deals with content-based image indexing and category retrieval in general databases. Statistical learning approaches have been recently introduced in CBIR. Labelled images are considered as training data in learning strategy based on classification process. We introduce an active learning strategy to select the most difficult images to classify with only few training data. Experimentations are carried out on the COREL database. We compare seven classification strategies to evaluate the active learning contribution in CBIR.
\end{abstract}

\section{INTRODUCTION}

Content-Based Image Retrieval (CBIR) has attracted a lot of research interest in recent years. This paper addresses the problem of category search, which aims at retrieving all images belonging to a given category from an image database.

Traditional techniques in CBIR are limited by the semantic gap, which separates the low-level information extracted from images and the semantic user request [20,9]: the user is looking for one image or an image set with semantics, for instance a type of landscape, whereas current processing deals with color or texture features. The increasing database sizes and the diversity of search types contribute to increase the semantic gap. Various strategies have been used to reduce the semantic gap.

Some off-line methods focus on the feature extraction or on the similarity function definition. In computer vision community, some works deal with local descriptor extraction [1, 24] and are concerned with creating indexes invariant to geometric transformations and robust to illumination changes. An image description may be built from local rotational invariant features and spatial constraints [22]. These models try to efficiently catch the visual structures of object categories. Thanks to psycho-visual experiments, Mojsilovic and Rogowitz [16] propose to identify image features and similarity functions which are directly connected to semantic categories. Experiments have also been carried out with user inter-

Permission to make digital or hard copies of all or part of this work for personal or classroom use is granted without fee provided that copies are not made or distributed for profi t or commercial advantage and that copies bear this notice and the full citation on the fi rst page. To copy otherwise, to republish, to post on servers or to redistribute to lists, requires prior specifi c permission and/or a fee.

$C V D B$ '04 Paris, France

Copyright 2004 ACM 1-58113-917-9/04/06 ...\$5.00. action to integrate a user model in a Bayesian similarity function [8]. The aim is to define a similarity between images as close as possible to the human similarity interpretation.

Other strategies focus on the on-line retrieval step to reduce the semantic gap. Interactive systems ask the user to conduct search within the database. Starting with a coarse query, the interactive process allows the user to refine that query as much as necessary. Most of the times, user provides binary annotations indicating whether or not the image belongs to the desired category. The system integrates these annotations through relevance feedback. Interactive retrieval techniques are mainly of two types: statistical and geometrical $[26,18]$. The geometrical methods refer to search-bysimilarity systems $[13,19]$. The objective of the statistical methods is to update a relevance function $[2,8]$ or a binary classification of images using the user annotations. Recently, statistical learning approaches have been introduced in CBIR context and have been very successful $[26,4]$. Discrimination methods (from statistical learning) may significantly improve the effectiveness of visual information retrieval tasks. This approach treats the relevance feedback problem as a supervised learning problem. A binary classifier is learn by using all relevant and irrelevant labelled images as input training data [5].

CBIR has a very specific classification context. There are very few training data during the retrieval process, the input space dimension is usually very high, unlabeled data are available, etc. Thus classical learning schemes have to be adapted. We analyze these specificities and propose some classification methods for comparison. We defend that active learning [7] may be helpful to carry out an efficient relevance feedback strategy. Active learning strategies offer a natural framework for interactive image retrieval and very efficient strategies, based on a SVM classification, have been proposed [23]. We introduce in this article an alternative to Tong's method, working as well with SVM classification as other classification methods. Our method, RETIN AL (RETIN Active Learning) is a new version of a previous search-by-similarity system, RETIN, working with both query and similarity updating [11]. Intensive experimentations are carried out on the COREL database. We compare seven classification strategies to evaluate the active learning contribution in CBIR.

\section{CLASSIFICATION METHODS}

The estimation of the searched category can be seen as a binary classification problem between relevant a class (1) and an irrelevant class (-1). In this section, three generative and discriminative learning methods, Bayes, kNN and SVM, are presented. They have 
been selected for their known classification performances in pattern recognition and CBIR context. To deal with non linearity of input data, all methods are kernelized. We denote Kernel functions by $k(.,$.$) .$

Notations: Let $\left(\mathbf{x}_{i}\right)_{i \in[1, N]}, \mathbf{x}_{i} \in \mathbb{R}^{p}$ be the feature vectors representing labelled images, and $\left(y_{i}\right)_{i \in[1, N]}, y_{i} \in\{-1,1\}$ be their respective annotations $(1=$ relevant, $-1=$ irrelevant $)$. We denote the relevance function, which returns the fellowship to the relevant class for any feature vector $\mathbf{x}$, by $f(\mathbf{x})$.

\subsection{Bayes Classifiers}

Bayes classifiers is used in text retrieval systems. Since ten years, CBIR community is transposing them to image retrieval $[25,26]$.

Bayes binary classifiers use the class-conditional likelihood associated with class $c P(\mathbf{x} \mid c)$ to compute the mapping function $g(\mathbf{x})$ of an input vector $\mathbf{x}$ :

$$
g(\mathbf{x})=\underset{c \in\{-1,1\}}{\operatorname{argmax}} P(\mathbf{x} \mid c) P(c)
$$

Because we have no prior assumption on the size of a class, we assume that $P(1)=P(-1)=\frac{1}{2}$. Once $g(\mathbf{x})$ is computed, the relevance function $f(\mathbf{x})$ may be expressed as follows:

$$
f(\mathbf{x})=P(\mathbf{x} \mid c=g(\mathbf{x}))
$$

To estimate the probability density function, we use a kernelized version of Parzen windows:

$$
P(\mathbf{x} \mid c)=\frac{1}{\left|\left\{i \mid y_{i}=c\right\}\right|} \sum_{i \in\left\{i \mid y_{i}=c\right\}} K\left(\mathbf{x}, \mathbf{x}_{i}\right)
$$

where $K(.,$.$) is a kernel function.$

\section{$2.2 k$-Nearest Neighbors}

This classification method has been used successfully in image processing and pattern recognition. For instance, in competition with neural networks, linear discriminant analysis (and others), kNearest Neighbors performed best results on pixel classification tasks (STATLOG project [15]).

$k$-Nearest Neighbors classifiers attempt to directly estimate $f(\mathbf{x})$ using only the $k$ nearest neighbors of $\mathbf{x}: f(\mathbf{x})=A v e\left(y_{i} \mid \mathbf{x}_{i} \in\right.$ $\left.n n_{k}(\mathbf{x})\right)$ where Ave denotes the average and $n n_{k}(\mathbf{x})$ the set of the $\mathrm{k}$ points nearest to $\mathrm{x}$ in squared distance. We use a kernelized version of these classifiers to better deal with non-linearity [12]:

$$
f(\mathbf{x})=\frac{\sum_{i \in n n_{k}(\mathbf{x})} y_{i} K\left(\mathbf{x}, \mathbf{x}_{i}\right)}{\sum_{i \in n n_{k}(\mathbf{x})} K\left(\mathbf{x}, \mathbf{x}_{i}\right)}
$$

\subsection{Support Vector Machines}

Support Vector Machines have shown their capacities in pattern recognition, and today know an increasing interest in CBIR [23, 5, $6,4]$.

The aim of SVM classification method is to find the best hyperplane separating relevant and irrelevant vectors maximizing the size of the margin (between both classes). Initial method assumes that relevant and irrelevant vectors are linearly separable. To overcome this problem, kernels $k(.,$.$) have been introduced. It allows to deal$ with non-linear spaces. Moreover, a soft margin may be used, in order to tolerate noisy configuration. It consists in a very simple

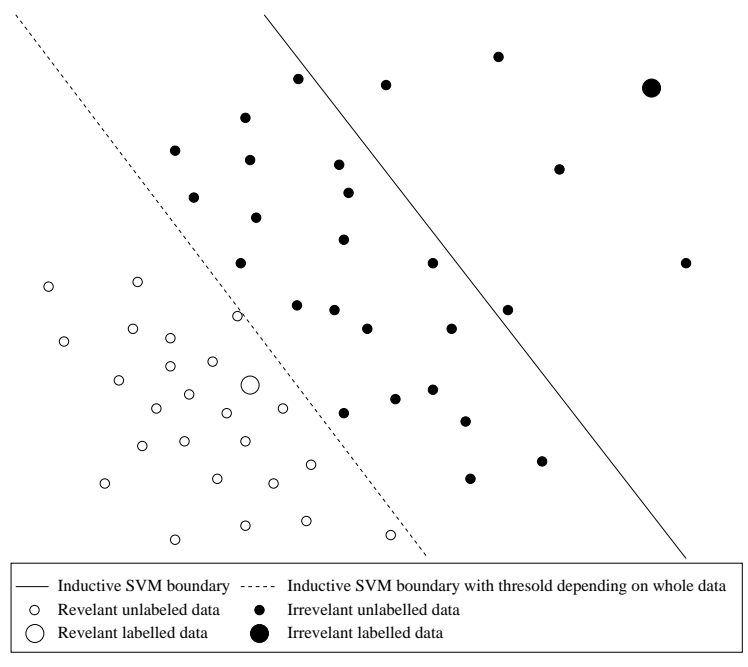

Figure 1: Little data artifact with SVM boundary.

adaptation by introducing a bound $C$ in the initial equations [27]. The resulting optimization problem may be expressed as follows:

$$
\begin{aligned}
& \boldsymbol{\alpha}^{\star}=\underset{\boldsymbol{\alpha}}{\operatorname{argmax}} \sum_{i=1}^{N} \alpha_{i}-\frac{1}{2} \sum_{i, j=1}^{N} \alpha_{i} \alpha_{j} y_{i} y_{j} K\left(\mathbf{x}_{i}, \mathbf{x}_{j}\right) \\
& \text { with }\left\{\begin{array}{l}
\sum_{\substack{i=1 \\
\forall}}^{N} \alpha_{i} y_{i}=0 \\
\forall i \in[1, N] \quad 0 \leq \alpha_{i} \leq C
\end{array}\right.
\end{aligned}
$$

Thanks to the optimal $\boldsymbol{\alpha}^{\star}$ value, the distance between a vector $\mathbf{x}$ and the separating hyperplane is used to evaluate how relevant is $\mathbf{x}$ :

$$
f(\mathbf{x})=\sum_{i=1}^{N} y_{i} \alpha_{i}^{\star} K\left(\mathbf{x}, \mathbf{x}_{i}\right)+b
$$

where $b$ is computed using the KKT Conditions [3].

\section{TRANSDUCTIVE METHODS}

In CBIR, systems have to classify image databases with very few training data. Meanwhile, all unlabeled images are available. If data are structured, unlabeled data should be useful for classification.

When very few labels are available, inductive SVM classification may have unexpected results. Fig. 1 shows such a case. Using only labelled data, the computed boundary is misplaced (full line). Many irrelevant data are misclassified. Such a configuration may happen when learning samples do not represent accurately the structure of data.

LeSaux[21] proposes to adapt the SVM scheme using unlabelled data. Only one parameter (threshold $b$ in Eq. 6) is modified for all the data. In the case of Fig. 1, this method provides a better classification (dotted line), but in the more complex case of Fig. 2, the boundary does not change.

Joachims proposes a method to deal with case of Fig. 2: Transductive SVM [14]. In this particular case, TSVM provides a good classification (dash dotted line). We adapt this approach, proposed in a text retrieval context. This method computes labels for unlabelled data such as hyperplane separates data with maximum margin. We used for experiments the $S V M_{\text {light }}$ implementation 
but on clusters of images: we compute $m$ clusters of images from $\mathbf{x}_{\left(s_{j}\right), j}$ to $\mathbf{x}_{\left(s_{j+M-1}\right), j}$ (where $M=10 \times m$ for instance), using an enhanced version of LBG algorithm [17]. Next, the system selects for labelling the most relevant image in each cluster. Thus, images close to each other in the feature space will not be selected together for labelling.

\section{RETIN AL SYSTEM FRAMEWORK}

RETIN AL (Active Learning) is a new version of the CBIR system developed in ETIS laboratory [11].

User interface is compound of two windows (Fig. 4). On top window, images in decreasing order of relevance are displayed, according the current classifier. On bottom window, images proposed for labelling are displayed, according to current active learner. The user is invited to follow advises in bottom window (best labelling according to current active learner), but he can choose to bypass these advises, and do some labelling of his own in the top window.

The user begins a new search with some images of his/her own. System updates the display in both windows. Next, user labels some pictures, and system updates the display, etc., until he/she is satisfied.

During experiment processes, the robot starts with some random pictures in the target category. During feedback, it acts as an user which always clicks in the bottom window.

\section{EXPERIMENTS}

\subsection{Feature Distributions}

Color and texture information are exploited. $L^{*} a^{*} b^{*}$ space is used for color, and Gabor filters, in twelve different scales and orientations, are used for texture analysis. Both spaces are clustered using an enhanced version of LBG algorithm [17]. We take the same class number for both spaces. Tests have shown that $c=25$ classes is a good choice for all our feature spaces [10]. Image signature is composed of one vector representing the image color and texture distributions. The input size $p$ is then 50 in our experiments.

\subsection{Database and evaluation protocol}

Tests are carried out on the generalist COREL photo database, which contains more than 50,000 pictures. To get tractable computation for the statistical evaluation, we randomly selected 77 of the COREL folders, to obtain a database of 6,000 images. To perform interesting evaluation, we built from this database 11 categories $^{1}$ ( $c f$. Table 1) of different sizes and complexities. The size of these categories varies from 111 to 627 pictures, and the complexity varies from monomodal (low semantics) to highly multimodal (high semantics) classes, relatively to feature vectors. Some of the categories have common images (for instance, castles and mountains of Europe, birds in savannah). For any category search, there is no trivial way to perform a classification between relevant and irrelevant pictures.

The CBIR system performances are measured using preci$\operatorname{sion}(\mathrm{P})$, recall $(\mathrm{R})$ and statistics computed on $\mathrm{P}$ and $\mathrm{R}$ for each category. Let us note $A$ the set of images belonging to the category, and $B$ the set of images returned to the user, then: $\mathrm{P}=\frac{|A \cap B|}{|B|}$ and $\mathrm{R}=\frac{|A \cap B|}{|A|}$. Usually, the cardinality of $B$ varies from 1 to database size, providing many points $(\mathrm{P}, \mathrm{R})$.

\footnotetext{
${ }^{1}$ A description of this database and the 11 categories can be found at: http://www-etis.ensea.fr/ cord/data/mcorel.tar.gz. This archive contains lists of image file names for all the categories.
}

\begin{tabular}{|c|c|c|}
\hline category & size & description \\
\hline birds & 219 & birds from all around the world \\
\hline castles & 191 & modern and middle ages castles \\
\hline caverns & 121 & inside caverns \\
\hline dogs & 111 & dogs of any species \\
\hline doors & 199 & doors of Paris and San Francisco \\
\hline Europe & 627 & European cities and countryside \\
\hline flowers & 506 & flowers from all around the world \\
\hline food & 315 & dishes and fruits \\
\hline mountains & 265 & mountains \\
\hline objects & 116 & single objects on an uniform background \\
\hline savannah & 408 & animals in African savannah \\
\hline
\end{tabular}

Table 1: COREL categories for evaluation

We use the average precision $P_{a}$ which represents the value of the $\mathrm{P} / \mathrm{R}$ integral function. This metric is used in the TREC VIDEO conference $^{2}$, and gives a global evaluation of the system (over all the $(\mathrm{P}, \mathrm{R})$ values).

\subsection{Comparative methods}

We evaluate seven methods:

- Three systems using the presented classification methods (Bayes, kNN and SVM) with a "basic" active learning algorithm: system presents to user to $m$ most relevant unlabelled images.

- Three systems using the presented classification method with the RETIN AL active learning algorithm.

- One system using SVM classification with Tong's active learning algorithm.

The kernel function used for SVM, kNN or Parzen estimation is a Gaussian kernel:

$$
K(x, y)=\exp ^{-\frac{1}{2 \sigma} d(x, y)^{2}}
$$

Moreover, the distance in Gaussian kernel may be chosen according to the feature vector type. We use a $\chi^{2}$ distance which is well suited for vectors representing distributions. As data is normalized, $\sigma$ is tuned to 1 .

When only one kind of labels is provided by user, binary classifications can not be computed. In this case, we use an estimation of the density of the labelled images to rank database, using a oneclass SVM method [6].

\subsection{Memory needs and computational com- plexity}

The main memory need is the storage of feature vectors ( $n p$ doubles) and kernel cache lines ( $n c$ doubles), where $n$ is the number of images in database, $p$ feature vector dimension, and $c$ the number of lines to cache. Other requirements are negligible against $n$. In the following experiments, about 3 Mo are used by features vectors, and 10 Mo for kernel cache (as many cache lines as the maximum of labels). With a one million image database, a similar configuration should require 400 Mo for feature vectors, and 1.6 Go for kernel cache.

The main computational needs is the $O(n)$ computation of fellowship to the relevant class (function $f($.$) in section 2$ ) on the whole database. Other requirements are negligible against $n$. In the following experiments, with a 10 Mo kernel cache, all methods need at most 2-3 seconds to compute with a Pentium $3 \mathrm{GHz}$. With a

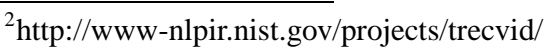




\begin{tabular}{|c|c|c|c|c|c|c|c|}
\hline category & Bayes/Basic & kNN/Basic & SVM/Basic & Bayes/RETIN & kNN/RETIN & SVM/RETIN & SVM/Tong \\
\hline birds & 19 & 29 & $\mathbf{3 1}$ & 20 & 29 & $\mathbf{3 1}$ & $\mathbf{3 1}$ \\
\hline castles & 15 & 17 & $\mathbf{3 8}$ & 17 & 18 & $\mathbf{3 8}$ & $\mathbf{3 8}$ \\
\hline caverns & 72 & 75 & 77 & 73 & 75 & $\mathbf{7 8}$ & 75 \\
\hline dogs & 22 & 28 & $\mathbf{5 8}$ & 21 & 32 & $\mathbf{5 8}$ & $\mathbf{5 8}$ \\
\hline doors & 86 & 88 & 89 & 91 & 90 & $\mathbf{9 3}$ & 83 \\
\hline Europe & 26 & 30 & 33 & 26 & 30 & $\mathbf{3 5}$ & $\mathbf{3 5}$ \\
\hline flowers & 56 & 59 & 60 & 64 & 63 & $\mathbf{6 7}$ & 57 \\
\hline food & 58 & 62 & 66 & 64 & 66 & $\mathbf{7 1}$ & 59 \\
\hline mountains & 30 & 42 & $\mathbf{5 4}$ & 39 & 39 & $\mathbf{5 4}$ & $\mathbf{5 4}$ \\
\hline objects & 60 & 69 & 75 & 67 & 67 & $\mathbf{7 8}$ & 76 \\
\hline savannah & 56 & 58 & 62 & 60 & 59 & $\mathbf{6 8}$ & 56 \\
\hline
\end{tabular}

Table 2: Performances: initialization with 1 relevant image, 5 annotations per feedback, 40 feedback steps.

\begin{tabular}{|c|c|c|c|c|c|c|c|}
\hline category & Bayes/Basic & kNN/Basic & SVM/Basic & Bayes/RETIN & kNN/RETIN & SVM/RETIN & SVM/Tong \\
\hline birds & 16 & 27 & $\mathbf{2 9}$ & 17 & 27 & $\mathbf{2 9}$ & $\mathbf{2 9}$ \\
\hline castles & 14 & 15 & $\mathbf{3 6}$ & 17 & 15 & $\mathbf{3 6}$ & $\mathbf{3 6}$ \\
\hline caverns & 70 & 74 & 77 & 72 & 74 & $\mathbf{7 8}$ & 75 \\
\hline dogs & 22 & 28 & $\mathbf{5 8}$ & 21 & 32 & $\mathbf{5 8}$ & $\mathbf{5 8}$ \\
\hline doors & 86 & 88 & 89 & 91 & 90 & $\mathbf{9 3}$ & 83 \\
\hline Europe & 26 & 30 & 33 & 26 & 30 & $\mathbf{3 5}$ & $\mathbf{3 5}$ \\
\hline flowers & 56 & 59 & 60 & 64 & 63 & $\mathbf{6 7}$ & 57 \\
\hline food & 58 & 62 & 66 & 64 & 66 & $\mathbf{7 1}$ & 59 \\
\hline mountains & 30 & 42 & $\mathbf{5 3}$ & 38 & 39 & $\mathbf{5 3}$ & $\mathbf{5 3}$ \\
\hline objects & 60 & 69 & 75 & 67 & 67 & $\mathbf{7 8}$ & 76 \\
\hline savannah & 56 & 58 & 61 & 59 & 59 & $\mathbf{6 7}$ & 55 \\
\hline
\end{tabular}

Table 3: Performances: initialization with 1 relevant image, 20 annotations per feedback, 10 feedback steps.

\begin{tabular}{|c|c|c|c|c|c|c|c|}
\hline category & Bayes/Basic & kNN/Basic & SVM/Basic & Bayes/RETIN & kNN/RETIN & SVM/RETIN & SVM/Tong \\
\hline birds & 24 & 33 & 36 & 24 & 33 & $\mathbf{3 8}$ & 34 \\
\hline castles & 15 & 25 & 40 & 16 & 27 & $\mathbf{4 1}$ & $\mathbf{4 1}$ \\
\hline caverns & 75 & 76 & 80 & 76 & 76 & $\mathbf{8 1}$ & 78 \\
\hline dogs & 39 & 50 & $\mathbf{6 4}$ & 42 & 50 & $\mathbf{6 4}$ & $\mathbf{6 4}$ \\
\hline doors & 85 & 89 & 90 & 91 & 90 & $\mathbf{9 3}$ & 83 \\
\hline Europe & 29 & 33 & 35 & 31 & 33 & $\mathbf{3 6}$ & $\mathbf{3 6}$ \\
\hline flowers & 59 & 62 & 65 & 66 & 66 & $\mathbf{6 9}$ & 59 \\
\hline food & 59 & 63 & 69 & 66 & 67 & $\mathbf{7 2}$ & 60 \\
\hline mountains & 49 & 46 & $\mathbf{5 5}$ & 50 & 47 & $\mathbf{5 5}$ & $\mathbf{5 5}$ \\
\hline objects & 71 & 74 & $\mathbf{8 3}$ & 75 & 74 & $\mathbf{8 3}$ & $\mathbf{8 3}$ \\
\hline savannah & 56 & 60 & 63 & 61 & 61 & $\mathbf{6 8}$ & 54 \\
\hline
\end{tabular}

Table 4: Performances: initialization with 11 relevant and 10 irrelevant images, 20 annotations per feedback, 9 feedback steps.

COREL evaluation: system performances estimated with the $P_{a}$ metric (\%), at the end of the interactive learning process. In all experiments, final training data sets exactly contain 201 images. 
[20] S. Santini, A. Gupta, and R. Jain. Emergent semantics through interaction in image databases. IEEE Transactions on Knowledge and Data Engineering, 13(3):337-351, 2001.

[21] B. Le Saux. Classification non exclusive et personnalisation par apprentissage : Application à la navigation dans les bases d'images. PhD thesis, INRIA, 2003.

[22] C. Schmid. Weakly supervised learning of visual models and its application to content-based retrieval. International Journal of Computer Vision, 56(1-2):7-16, January,February 2004.

[23] S. Tong and E. Chang. Support vector machine active learning for image retrieval. In ACM Multimedia, 2001.

[24] T. Tuytelaars and L. Van Gool. Content-based image retrieval based on local affinely invariant regions. In Third Int'l Conf. on Visual Information Systems, Visual99, pages 493-500, 1999.

[25] N. Vasconcelos. Bayesian models for visual information retrieval. $\mathrm{PhD}$ thesis, Massachusetts Institute of Technology, 2000.

[26] N. Vasconcelos and M. Kunt. Content-based retrieval from image databases: current solutions and future directions. In International Conference in Image Processing (ICIP'01), volume 3, pages 6-9, Thessaloniki, Greece, October 2001.

[27] K. Veropoulos. Controlling the sensivity of support vector machines. In International Joint Conference on Artificial Intelligence (IJCAI99), Stockholm, Sweden, 1999.

[28] X. Zhu, J. Lafferty, and Z. Ghahramani. Combining active learning and semi-supervised learning using gaussian fields and harmonic functions. In ICML 2003 workshop on The Continuum from Labeled to Unlabeled Data in Machine Learning and Data Mining, 2003. 